\title{
Combined endoscopic third ventriculostomy and choroid plexus cauterization as primary treatment for infant hydrocephalus: a prospective North American series
}

\author{
Clinical article
}

\author{
Scellig S. D. Stone, M.D., Ph.D., and Benjamin C. Warf, M.D. \\ Department of Neurosurgery, Boston Children's Hospital, Boston, Massachusetts
}

\begin{abstract}
Object. Combined endoscopic third ventriculostomy with choroid plexus cauterization (ETV/CPC) enhances the likelihood of shunt freedom over ETV alone, and thus avoidance of shunt-related morbidity, in hydrocephalic infants. To date, virtually all published reports describe experiences in Africa, thus hampering generalization to other parts of the world. Here, the authors report the first North American prospective series of this combined approach to treat hydrocephalus of various etiologies in infants.

Methods. A prospective series of 50 boys and 41 girls (mean and median ages 4.7 and 3.2 months, respectively) with hydrocephalus underwent ETV/CPC performed by the senior author at Boston Children's Hospital from August 2009 through March 2014. Success data were analyzed using the Kaplan-Meier method and Cox proportional hazards model.

Results. The 91 patients treated included those with aqueductal stenosis (23), myelomeningocele (23), posthemorrhagic hydrocephalus (25), Dandy-Walker complex (6), post-infectious hydrocephalus (6), and other conditions (8). Using Kaplan-Meier survival analysis, 57\% of patients required no further hydrocephalus treatment at 1 year. Moreover, $65 \%$ remained shunt free to the limit of available follow-up (maximum roughly 4 years). A Cox proportional hazards model identified the following independent predictors of ETV/CPC failure: post-infectious etiology, age at treatment younger than 6 months, prepontine cistern scarring, and prior CSF diversion. Of patients with at least 6 months of follow-up, the overall ETV/CPC success at 6 months (59\%) exceeded that predicted by the ETV Success Score (45\%). Complications included 1 CSF leak and 1 transient syndrome of inappropriate antidiuretic hormone secretion, and there were no deaths.

Conclusions. ETV/CPC is an effective, safe, and durable treatment for infant hydrocephalus in a North American population, with 1-year success rates similar to those reported in Africa and equivalent to those for primary shunt placement in North America. These findings underscore the need for prospective multicenter studies of the outcomes, quality of life, and economic impact of the procedure compared with primary shunt insertion. (http://thejns.org/doi/abs/10.3171/2014.7.PEDS14152)
\end{abstract}

\section{Key Words • hydrocephalus - third ventriculostomy - endoscopy • choroid plexus cauterization}

I N 2005, Warf first reported the technique and results of combining endoscopic third ventriculostomy (ETV) with bilateral endoscopic lateral ventricle choroid plexus cauterization (CPC) to treat hydrocephalus in infants. ${ }^{13}$ Subsequently, the efficacy of the procedure has been demonstrated among distinct etiologies of hydrocephalus in infants, with overall long-term success of more than $60 \%$ for all patients if the prepontine cistern is not obstructed by arachnoid scarring. ${ }^{14,16-19,22,23}$ With the exception of 2 studies from US institutions of ETV/CPC

\footnotetext{
Abbreviations used in this paper: $\mathrm{CPC}=$ choroid plexus cauterization; ETV = endoscopic third ventriculostomy; ETVSS = ETV Success Score; $\mathrm{MMC}=$ myelomeningocele; $\mathrm{PHH}=$ post-hemorrhagic hydrocephalus; $\mathrm{PIH}=$ post-infectious hydrocephalus; $\mathrm{VP}=$ ventriculoperitoneal.
}

for post-hemorrhagic hydrocephalus $(\mathrm{PHH})$ of prematurity, ${ }^{2,17}$ there have been no reports to date in the Englishlanguage literature of the overall effectiveness of the procedure for infants outside of Africa. Here, we report the results of the first consecutive series of ETV/CPC as primary treatment for hydrocephalus of all etiologies in infants from a North American center.

\section{Methods}

\section{Patient Population}

With approval of the Boston Children's Hospital in-

This article contains some figures that are displayed in color online but in black-and-white in the print edition. 
stitutional review board, data were prospectively collected from all procedures performed for definitive treatment of hydrocephalus by the senior author at Boston Children's Hospital. From August 2009 through March 2014, 100 consecutive children younger than 24 months were treated. Of these, 93 electively underwent ETV/CPC, and in $91(98 \%)$ the procedure was completed. In 1 patient with $\mathrm{PHH}$ the ETV/CPC procedure was abandoned for reservoir placement due to turbid CSF and poor visibility. In another infant with PHH a ventriculoperitoneal (VP) shunt was placed because of significant scarring in the prepontine cistern discovered after performing the ETV. Seven patients were purposely not offered ETV/CPC as a treatment option. Four of these patients were infants with PHH who underwent primary shunt placement because preoperative sagittal FIESTA MRI demonstrated obstruction of the prepontine cistern. We have previously reported this condition as a significant predictor of ETV/ CPC failure for infants with PHH and post-infectious hydrocephalus (PIH). ${ }^{17,20}$ One patient with congenital aqueductal stenosis underwent primary VP shunt placement because preoperative MRI showed that the third ventricle floor was overriding the sella turcica, which prohibited ETV from being safely performed. One infant with PHH presented immediately to the emergency department with papilledema and MRI showing robust flow through the aqueduct and the fourth ventricle outlet foramina, and a decision was made to urgently place a VP shunt. One 22-month-old infant with PHH and a prior VP shunt underwent ETV without CPC earlier in the series before $\mathrm{CPC}$ was routinely being offered to children older than 12 months. The other 6 patients in the series who were older than 12 months underwent ETV/CPC.

The database was then retrospectively verified for accuracy by chart review. If an individual patient underwent additional ETV procedures, only data from the first procedure were included in our analysis. The 91 patients who underwent completed ETV/CPC were included in this analysis exclusively. The mean and median uncorrected ages at treatment were 4.7 and 3.2 months, respectively. There were 41 girls and 50 boys.

\section{Surgical Procedure and Data Collection}

The operative methods used were very similar to those reported previously..$^{13}$ All patients underwent an ETV via a unilateral frontal trajectory using a flexible endoscope (model 11282 BN1 3.7 mm in diameter, Karl Storz), Xenon light source, and display monitor. A small semilunar incision was made at the lateral corner (typically on the right) of the anterior fontanel, centered in the midpupillary line. The use of electrocautery on the scalp was minimized, and the small curved flap was retracted with a stitch. A linear 4-mm dural incision without the use of electrocautery was made to facilitate a watertight dural closure. Fenestration of the floor of the third ventricle and/or the lamina terminalis was performed using a Bugby electrocautery wire without cautery, and the opening was variably dilated using a $0.7-\mathrm{mm}$ balloon catheter filled with irrigation solution or by stretching of the opening with the wire by flexing and torquing the endoscope tip. Additional prepontine obstructive membranes (such as the Liliequist membrane) were similarly fenestrated when present. Visual inspection of the prepontine cistern and/or lamina terminalis cistern was performed after fenestration of the third ventricle, and the findings were qualitatively recorded at the time of surgery as either scarred or not scarred. We qualitatively defined a scarred cistern as abnormally thickened and opaque arachnoid that either partially or completely obstructed the cistern. When appropriate, these membranes were dissected through using the Bugby wire and balloon catheter to open a pathway into the open cistern more distally. Aqueduct patency was determined by visual inspection of the aqueduct ostium. This inspection helped distinguish whether aqueducts that appeared very stenotic on preoperative MRI were truly obstructed or simply narrow. The aqueduct was categorized as closed or not closed (for example, a stenotic ostium was considered not closed). The lateral ventricle choroid plexus was cauterized bilaterally from the foramen of Monro to its anterior terminus in the tip of the temporal horn using a Bugby wire. If necessary, a septostomy was performed to gain access to the contralateral lateral ventricle.

We defined ETV/CPC treatment success as the absence of any subsequent surgical procedure for hydrocephalus treatment (including successful reoperation to open a closed ETV) or death related to hydrocephalus management. Standard treatment failure criteria, as for a shunt failure, were used to determine whether further surgery was required: a greater than normal rate of growth in head circumference as plotted on a standard growth chart, anterior fontanel distension, new or worsening symptoms of elevated intracranial pressure, new or worsening abnormal eye findings, or progressive ventriculomegaly on cranial imaging. Patients typically underwent follow-up at 2 weeks and 1, 3, 6, and 12 months after the procedure with cranial ultrasonography and head circumference measurements performed at each visit. In cases in which fontanel patency was insufficient to allow ultrasonography, rapid sequence "ventricle check" T2-weighted MRI was used to assess the ventricles. A full postoperative MRI study, including sagittal T2-weighted SPACE or FIESTA images, was typically obtained at 6 months and at 1 year after surgery to assess evidence for ETV patency and to obtain detailed measurements for frontal-occipital horn ratio determination.

\section{Statistical Analysis}

ETV/CPC success data were analyzed using the Kaplan-Meier method. Estimates of hazard ratios for the time to ETV/CPC failure were derived using a Cox proportional hazards model. A single multivariable model was used to test several planned independent categorical variables: etiology of hydrocephalus (aqueductal stenosis, Dandy-Walker complex, myelomeningocele [MMC], $\mathrm{PIH}, \mathrm{PHH}$, or other), age at treatment $(<183$ days or $\geq$ 183 days), prior CSF diversion (prior diversion or no prior diversion), presence of cistern scarring (scarred or not scarred), and aqueduct patency (closed or not closed). Variance inflation factors for all independent variables were $<1.2$, negating potential concerns for multicollinearity. The appropriateness of the proportional hazards assumption was verified by examining stratified survival 
curves for each independent variable. Frequencies of categorical variables significantly associated with ETV/CPC success were compared between etiologies using Fisher's exact tests. Predicted success with ETV alone (without CPC) at 6 months was determined using the ETV Success Score (ETVSS). ${ }^{5}$ Predicted ETV success and actual ETV/CPC success at 6 months were then compared using a 2-tailed paired t-test. Analyses were performed using SPSS Statistics (version 21, IBM Inc.) and Prism 5 (GraphPad Software Inc.).

\section{Results}

Ninety-one patients (50 boys and 41 girls) underwent ETV/CPC as the primary treatment for hydrocephalus. Two of these patients had only unilateral CPC when limited visibility precluded safely proceeding into the contralateral lateral ventricle. The uncorrected age at treatment ranged from 2 to 701 days with mean and median ages of 4.7 and 3.2 months, respectively. Of 91 patients, 84 (92\%) were younger than 1 year. The predominant causes of hydrocephalus were MMC (23), PHH (25), and aqueductal stenosis (23), with each comprising slightly more than onefourth of the study group. The remaining one-fourth comprised Dandy-Walker complex (6), PIH (6), congenital idiopathic hydrocephalus (5), and congenital cyst (3). Two of the infants with congenital cysts had agenesis of the corpus callosum, an interhemispheric fissure cyst, and severe congenital hydrocephalus. The third patient had a large middle fossa and quadrigeminal cistern cyst that compressed the aqueduct; initial cyst fenestration alone failed, and the patient went on to have ETV/CPC in combination with repeat cyst fenestration. There were no infections and no deaths. Complications included 1 CSF leak and 1 transient syndrome of inappropriate antidiuretic hormone secretion.

Figure 1 depicts the overall Kaplan-Meier ETV/CPC success analysis for the 91 patients treated. Kaplan-Meier analysis estimated ETV/CPC success rates at 6 and 12 months were $65 \%$ and $57 \%$, respectively. The median follow-up was 18.6 months. Characteristics included in the Cox regression model and their respective hazard ratios are shown in Table 1. ETV/CPC was much less successful for postmeningitic hydrocephalus than for the other etiologies, which otherwise had similar success regardless of etiology (Fig. 2). Consistent with previous reports, age 6 months and older (Fig. 3), no prior CSF diversion (Fig. 4), and lack of cistern scarring (Fig. 5) were all associated with a substantially better chance of ETV/CPC success (HRs 0.21-0.29). We had further stratified outcome according to ages 0 to $<3,3$ to $<6,6$ to $<9$, and $9-12$ months. There was no significant difference in outcome among any of these age groups younger than 6 months or any of the age groups 6 months or older (Fig. 6). Thus, we found the age of 6 months to be an important watershed as opposed to a more gradual progression with age. Aqueduct patency was not associated with treatment success $(p=0.13)$ and was therefore removed from the model. Reopening of an obstructed ETV was attempted in 12 patients, and while the treatment in these patients was considered to have failed for the purpose of statistical analyses, 3 patients (25\%) have remained shunt free.

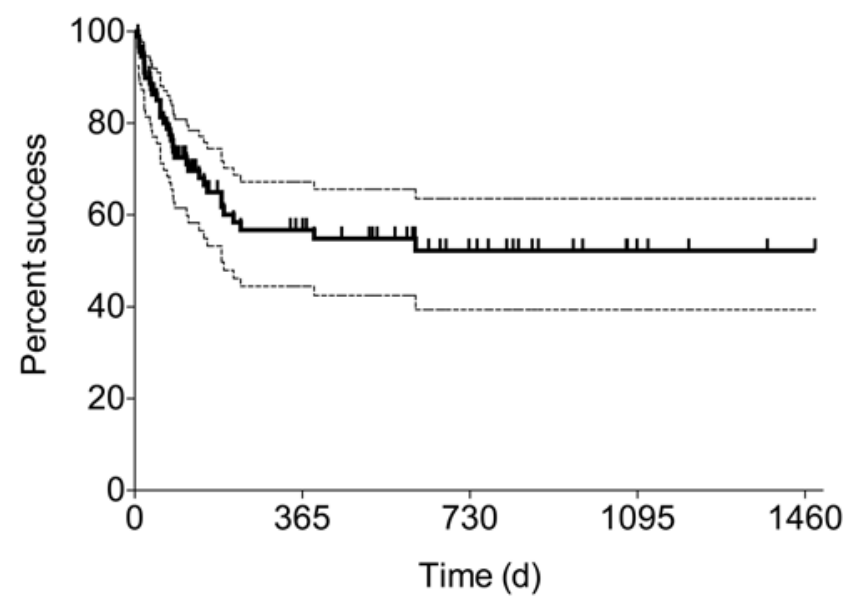

FIg. 1. Kaplan-Meier-derived ETVICPC success curve (with 95\% Cls) for all patients. $d=$ days.

The proportion of patients with no prior CSF diversion (Table 2) varied according to etiology ( $p<0.01$, Fisher's exact test), with most cases of prior diversion found in the PHH group. However, the hazard of prior diversion appeared similar across etiologies. Specifically, the addition of an interaction term between etiology and prior diversion to the Cox regression model was not significant $(\mathrm{p}=0.14)$. In addition, the $\mathrm{HR}$ for prior diversion remained significant $(\mathrm{p}=0.03)$ and of similar magnitude (0.26 [95\% CI 0.10-0.87]) when the analysis was stratified by etiology.

The proportion of patients with clean cisterns (Table 2) also varied according to etiology $(p=0.01$, Fisher's exact test), with the majority of scarred cisterns encountered in either the PIH or PHH groups. Adding an interaction term between etiology and cistern to the Cox regression model produced significance $(\mathrm{p}=0.01)$; however, there were fewer than 5 events per predictor variable for some etiologies and thus an elevated risk of Type 1 error. In contrast, the HR for cistern remained significant $(\mathrm{p}=$ 0.04 ) and of similar magnitude (0.39 [95\% CI 0.15-0.99]) when the analysis was stratified by etiology. Given the limited sample size and stratified results, a robust difference in the effect of cistern scarring across different etiologies could not be demonstrated; however, cistern scarring is not an expected feature of noninfectious and nonhemorrhagic etiologies of hydrocephalus. Our prior work examining PIH with greater statistical power reported an association between lack of cistern scarring and ETV/CPC success, ${ }^{20}$ and our initial study of ETV/ CPC in those with $\mathrm{PHH}$ demonstrated the same result. ${ }^{17}$ A planned comparison in this study within the relatively large $\mathrm{PHH}$ group (Fig. 7) demonstrated a similar trend (HR 0.38 [95\% CI 0.09-1.57]).

The proportion of patients 6 months and older did not vary according to etiology ( $p=0.25$, Fisher's exact test) and thus the effect of age on ETV/CPC success appeared consistent regardless of etiology.

Of the 69 patients having at least 6 months of followup, the overall ETV/CPC success at 6 months was 59\% (95\% CI $48 \%-71 \%$ ). This exceeded the $45 \%$ (95\% CI $41 \%-$ $49 \%$ ) success predicted for ETV alone using the ETVSS (p 
TABLE 1: Summary of patient characteristics included in Cox regression model and test results

\begin{tabular}{|c|c|c|c|c|c|}
\hline Characteristic & $\begin{array}{c}\text { No. of } \\
\text { Patients (\%) }\end{array}$ & $\begin{array}{l}\text { No. of Patients Needing } \\
\text { Subsequent Procedure }\end{array}$ & $\begin{array}{l}\text { Median Time to Failure } \\
\text { (days) }\end{array}$ & $\begin{array}{l}\text { HR for ETV/CPC Failure } \\
(95 \% \mathrm{Cl})\end{array}$ & $p$ Value \\
\hline \multicolumn{6}{|l|}{ etiology } \\
\hline aqueductal stenosis & $23(25.3)$ & 9 & not reached & $0.14(0.04-0.44)$ & $<0.01$ \\
\hline Dandy-Walker complex & $6(6.6)$ & 3 & 776 & $0.61(0.14-2.66)$ & 0.51 \\
\hline MMC & $23(25.3)$ & 8 & not reached & $0.13(0.04-0.46)$ & $<0.01$ \\
\hline $\mathrm{PIH}$ & $6(6.6)$ & 5 & 21 & reference (1) & \\
\hline $\mathrm{PHH}$ & $25(27.5)$ & 7 & not reached & $0.06(0.02-0.25)$ & $<0.01$ \\
\hline other & $8(8.8)$ & 3 & not reached & $0.56(0.12-2.67)$ & 0.46 \\
\hline \multicolumn{6}{|l|}{ age at treatment } \\
\hline$<183$ days & $62(68.1)$ & 29 & 215 & reference (1) & \\
\hline$\geq 183$ days & $29(31.9)$ & 6 & not reached & $0.21(0.08-0.59)$ & $<0.01$ \\
\hline \multicolumn{6}{|l|}{ prior CSF diversion } \\
\hline prior diversion & $17(18.7)$ & 8 & 231 & reference (1) & \\
\hline no prior diversion & $74(81.3)$ & 27 & not reached & $0.24(0.08-0.66)$ & 0.01 \\
\hline \multicolumn{6}{|l|}{ cistern scarring } \\
\hline scarred & $15(16.5)$ & 8 & 87 & reference (1) & \\
\hline not scarred & $76(83.5)$ & 27 & not reached & $0.29(0.11-0.76)$ & 0.01 \\
\hline
\end{tabular}

$=0.02$, 2-tailed paired t-test). Moreover, the rate of ETV/ $\mathrm{CPC}$ success beyond 6 months remained greater than the 6-month success predicted by the ETVSS and to the limit of available follow-up. At 6 months, the rate of successful treatment by ETV/CPC in the 62 patients without postmeningitic hydrocephalus, a scarred prepontine cistern, or a previous shunt was $67 \%$ for those younger than 6 months and $79 \%$ for those older than 6 months (Fig. 8).

\section{Discussion}

In 2005, Warf reported the initial experience in Uganda with treating hydrocephalus in infants using a combination of both ETV and CPC (Fig. 9)..$^{13}$ In that study, for all infants younger than 12 months, ETV/CPC was successful with no further operations in $66 \%$ in comparison with $47 \%$ success with ETV alone $(\mathrm{p}<0.0001)$.

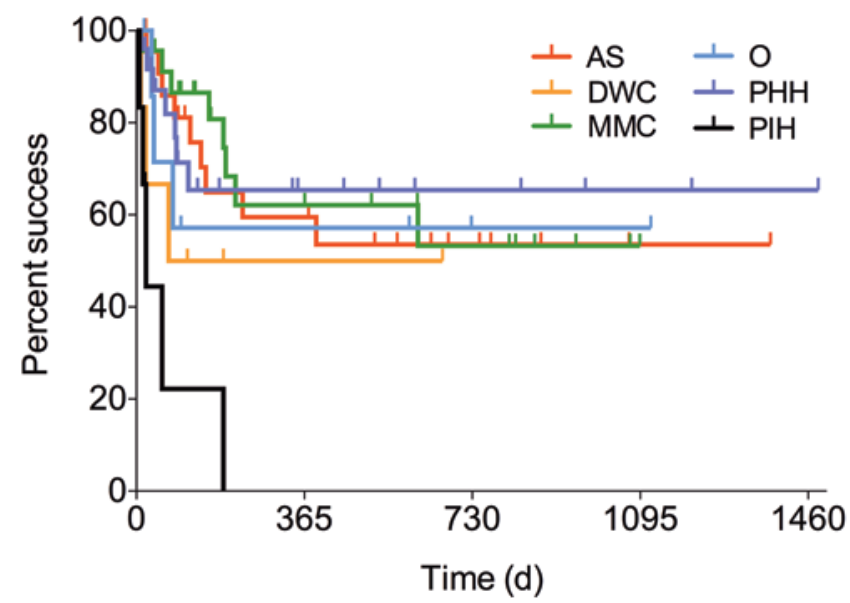

FIG. 2. Kaplan-Meier derived ETVICPC success curves by etiology. $\mathrm{AS}=$ aqueductal stenosis; DWC = Dandy-Walker complex; $\mathrm{O}=$ other.
The efficacy of the combined procedure was subsequently reported in a variety of individual etiologies, including MMC, encephalocele, aqueductal stenosis, PIH, DandyWalker complex, and congenital idiopathic hydrocephalus of infancy. ${ }^{14,16-19,22,23}$

The patient populations in Uganda and the US are different in some important respects. First, the most common cause of infant hydrocephalus in Uganda is neonatal ventriculitis, rare in North America, for which the pathogenesis remains obscure. , $^{4,9,15}$ By contrast, as demonstrated in the present series, one of the most common causes of infant hydrocephalus in North America is that resulting from intraventricular hemorrhage of prematurity, rare in Uganda because very premature infants generally do not survive. However, there are similarities between ventriculitis and intraventricular hemorrhage in that the final common mechanism of hydrocephalus is the

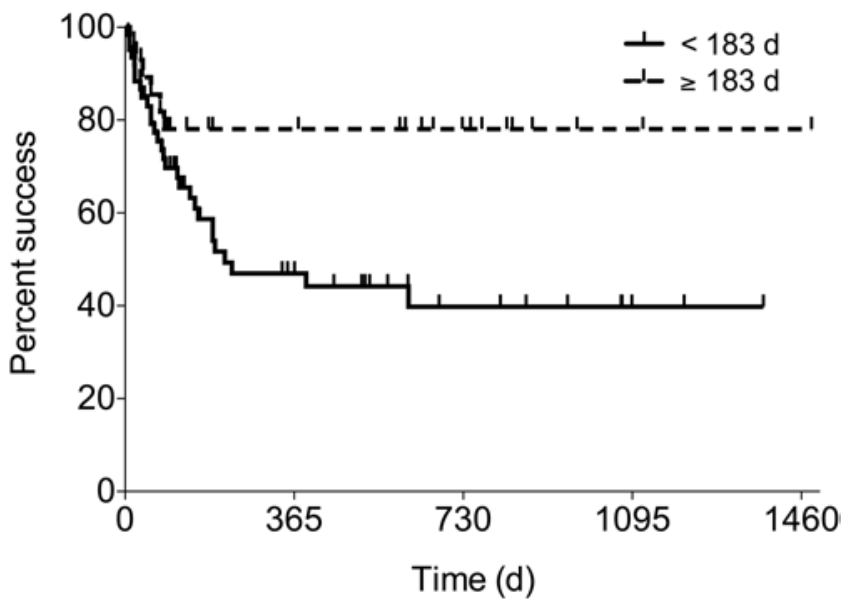

FIG. 3. Kaplan-Meier derived ETV/CPC success curves by age at treatment.

J Neurosurg: Pediatrics / Volume 14 / November 2014 


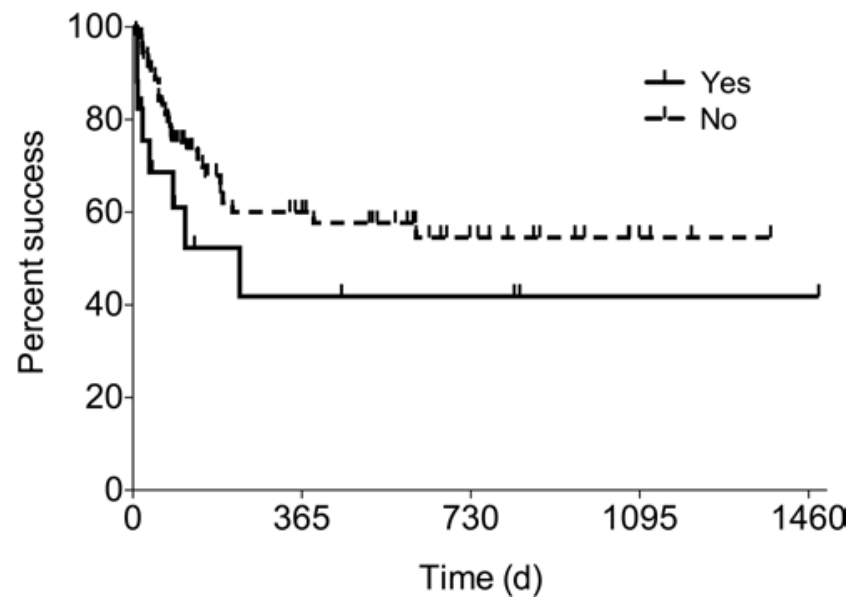

FIG. 4. Kaplan-Meier derived ETV/CPC success curves by prior CSF diversion.

inflammatory response, with intraventricular ependymal scarring leading to obstruction at the level of the foramina of Monro, aqueduct, or fourth ventricular outlets, and with variable involvement of the subarachnoid spaces. We previously demonstrated that scarring in the prepontine cistern was a significant risk factor for ETV/CPC failure in PIH in Uganda, and for PHH in the US. ${ }^{17,20}$ The latter observation has recently been corroborated by others. ${ }^{2}$

Another contrast between these patient populations is the time of presentation, with a longer interval between onset and treatment for Ugandan infants. The mean and median ages at treatment in the Ugandan study were 14 months and 5 months, respectively. ${ }^{13}$ By contrast the mean and median ages were 4.7 and 3.2 months, respectively, in the current study. Thus, the current series represents a comparatively younger group. Other differences in Uganda compared with the US include the lack of preoperative MRI in Uganda and the greater difficulty in patient follow-up. These factors, and the addition of CPC to the ETV procedure, invalidated the use of the ETVSS in the Ugandan population and prompted our creation of a unique Uganda ETVSS that took CPC into account. .,21 $^{2}$

Given the differences between the populations, it was

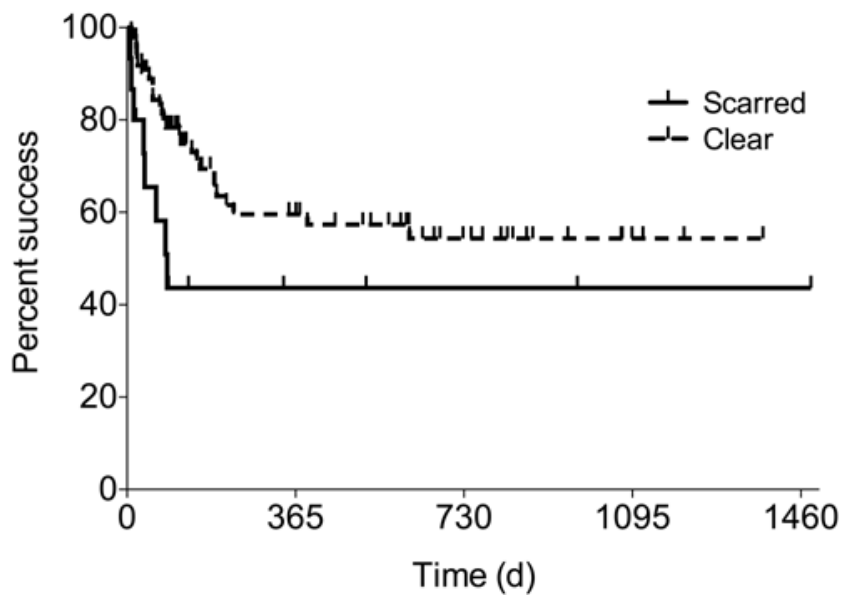

FIG. 5. Kaplan-Meier derived ETVICPC success curves by cistern scarring.

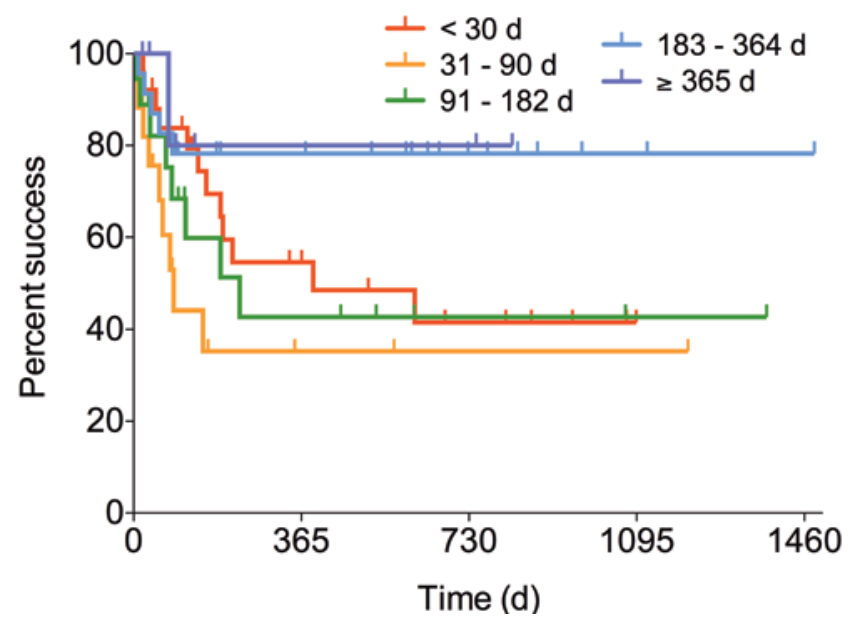

FIG. 6. Kaplan-Meier derived ETV/CPC success curves by age at treatment.

not known whether the ETV/CPC procedure would yield results in North America that were similar to those reported from Uganda. We had demonstrated the efficacy of sagittal FIESTA MRI in predicting severe prepontine scar in patients with $\mathrm{PHH}$ and reported in a small series that the results for infants having no significant prepontine arachnoid scar was similar to that for Ugandan infants with PIH. ${ }^{17,20}$ Since there should be no intrinsic difference in outcome between Ugandan and American infants with congenital etiologies of hydrocephalus, we hypothesized that the overall results would be similar.

The study reported here is limited by the small number of patients having any particular etiology of hydrocephalus. As expected, most of the patients had hydrocephalus due to $\mathrm{PHH}, \mathrm{MMC}$, or congenital aqueductal stenosis, each comprising about $25 \%$ of the cases. The success of ETV/CPC in each of these groups was similar. The results for postmeningitic hydrocephalus were disappointing; however, they accounted for only 6 patients (7\%) in this series. In addition, the North American cases were different from the PIH patients in Uganda, in that there was much less evidence of significant intraventricular inflammation. The PIH etiology described for Uganda has a significant ventriculitis component and is commonly accompanied by parenchymal destruction. In addition, the bacterial etiology of the ventriculitis in Ugandan infants has been difficult to identify in contrast to the organisms

TABLE 2: Proportions of patients with no prior CSF diversion and clean cisterns by etiology

\begin{tabular}{lccc}
\hline $\begin{array}{c}\text { Hydrocephalus } \\
\text { Etiology }\end{array}$ & $\begin{array}{c}\text { No. of } \\
\text { Patients }\end{array}$ & $\begin{array}{c}\text { No. of Patients } \\
\text { w/ No Prior CSF } \\
\text { Diversion (\%) }\end{array}$ & $\begin{array}{c}\text { No. of Patients } \\
\text { w/ Unscarred } \\
\text { Cisterns (\%) }\end{array}$ \\
\hline aqueductal stenosis & 23 & $21(91.3)$ & $21(91.3)$ \\
Dandy-Walker complex & 6 & $6(100)$ & $6(100)$ \\
MMC & 23 & $21(91.3)$ & $23(100)$ \\
PIH & 6 & $6(100)$ & $3(50.0)$ \\
PHH & 25 & $13(52.0)$ & $16(64.0)$ \\
other & 8 & $8(100)$ & $7(87.5)$ \\
\hline
\end{tabular}




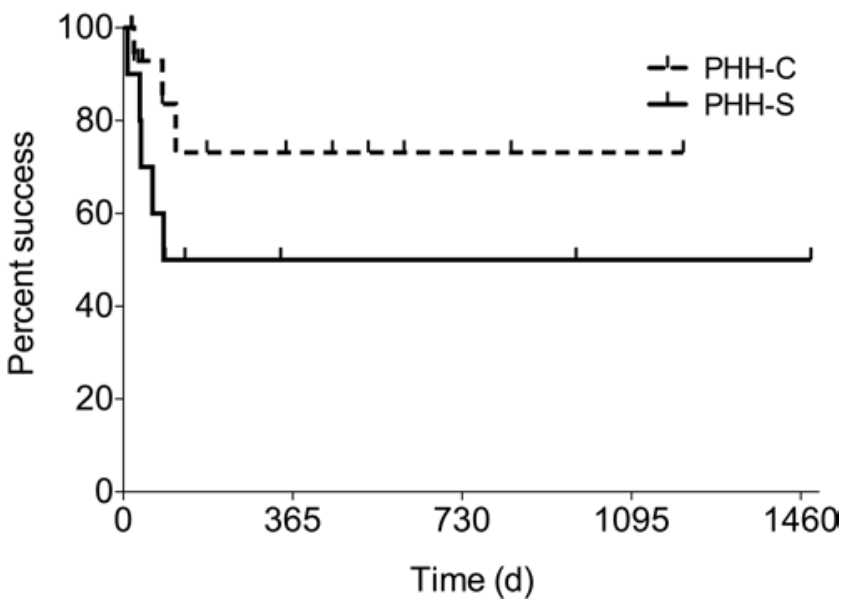

FIG. 7. Kaplan-Meier derived ETV/CPC success curves in the PHH group by cistern scarring. $\mathrm{C}=$ clear cistern; $\mathrm{S}=$ scarred cistern.

common to infant meningitis in the US. ${ }^{4,7,9,15}$ Based on the results in the present study, we currently do not recommend ETV/CPC for postmeningitic hydrocephalus in our North American practice.

Overall, with 3 successful redo ETVs, 59 (65\%) of 91 patients undergoing ETV/CPC remained shunt free. It is notable that in this nonselected series of 100 consecutive children younger than 2 years presenting for treatment of hydrocephalus, 59 (59\%) were successfully treated without a shunt. Of the 3 most common etiologies for infant hydrocephalus presenting for treatment at prior to 6 months of age and without having had a prior VP shunt placed, shunt freedom has been maintained in 8 (62\%) of 13 with aqueductal stenosis (including one successful repeat ETV), 7 (88\%) of 8 for $\mathrm{PHH}$ with no prior ventriculosubgaleal shunt, and $11(69 \%)$ of 16 including those with prior ventriculosubgaleal shunt. In regard to MMC patients treated prior to 6 months of age, $14(74 \%)$ of 19 (including 1 who underwent a successful redo ETV) remained shunt free. Two of the MMC patients who experienced failure underwent shunt treatment due to stridor did not benefit from shunting and went on to have a Chiari decompression. If these 2 cases are not counted as ETV/

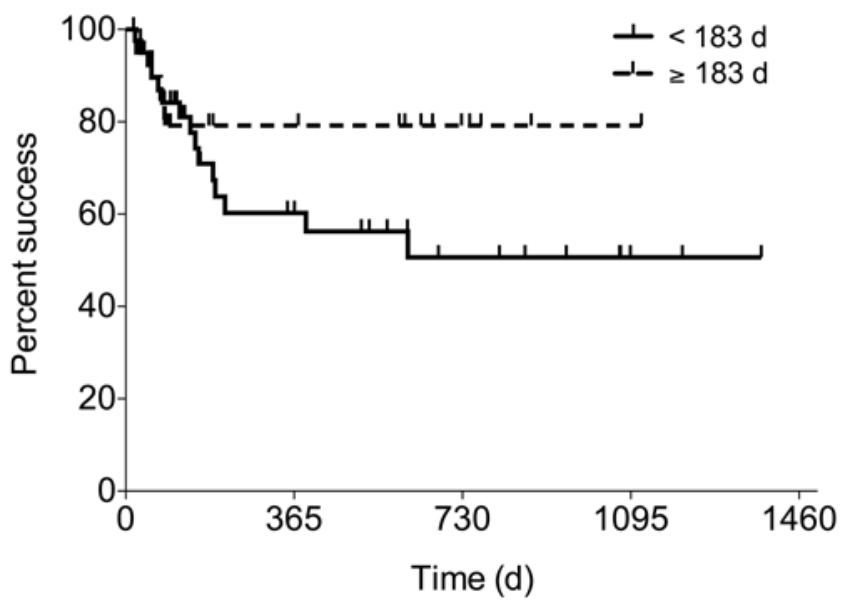

FIG. 8. Kaplan-Meier derived ETV/CPC success curves excluding $\mathrm{PIH}$, prior CSF diversion, and scarred cistern by age.

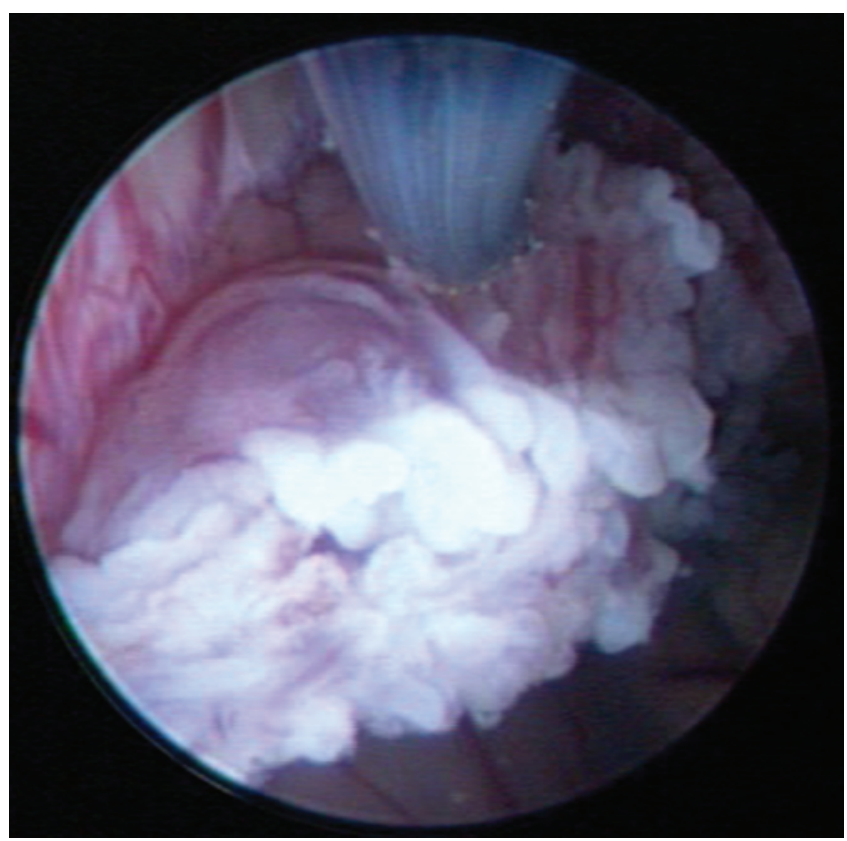

FIG. 9. Ventriculoscopic image of CPC with a Bugby wire.

CPC failures (since shunting was not otherwise clearly indicated and did not resolve the symptoms), then the success for MMC infants younger than 6 months would be 16 $(84 \%)$ of 19 , which is similar to that reported previously in the larger series from Uganda. ${ }^{16}$

It is notable that, in the current study, the predicted success of ETV by the ETVSS was $45 \%$ while the actual overall success for ETV in the Uganda series was $47 \%$, and the overall ETV/CPC success in the current study was $59 \%$ compared with $66 \%$ in the Ugandan series. ${ }^{13}$ Thus, the overall success of ETV/CPC in the current study is close to that previously reported from the larger Ugandan study.

In the Ugandan ETV success prediction score, the most heavily weighted factor was the degree of CPC. ${ }^{21}$ This suggests that outcome is dependent upon technique, and this will need to be accounted for in future studies by other investigators. Except for cases of extremely severe ventriculomegaly, the choroid plexus of the lateral ventricles cannot be fully accessed with a rigid endoscope. There is typically a significant volume of choroid plexus from the root to the tip of the temporal horn that can only be accessed safely using flexible ventriculoscopy, and this requires a somewhat different skill set. The fact that CPC "levels the playing field" for ETV among different etiologies and ages is demonstrated by the similar success rates among the various etiologies in the present study, with the important exception of hydrocephalus secondary to meningitis, and the observation that outcomes are superior to those predicted by the ETVSS.

Brain development is an important measure of true success in the treatment of hydrocephalus. As in other studies, however, success of endoscopic hydrocephalus treatment here was defined by the same parameters typically used to define successful shunt treatment, without taking neurocognitive development into account. As yet, there are no published results from prospective controlled 
trials to establish whether there is any difference between treatment modalities in regard to developmental outcome. In a retrospective study, we previously found no difference in developmental outcome when comparing the 2 treatments in patients with hydrocephalus associated with MMC, ${ }^{12}$ and similar findings were subsequently reported by others. ${ }^{11}$ This study also demonstrated no correlation between ventricle size and developmental outcome. A secondary analysis of the same population has suggested the combined assessment of brain and CSF volumes as a predictor of developmental outcome, and there was no difference in this parameter between patients treated by shunt placement and those treated by ETV/CPC. ${ }^{8}$ Randomized prospective trials of treatment by shunt placement versus ETV/CPC in etiologically homogeneous infant hydrocephalus populations that measure developmental outcome and brain and CSF volumes are needed to look for meaningful differences between these treatment modalities. We are currently conducting such a study in Ugandan infants with PIH.

That no difference in early childhood development was found between MMC infants treated by ETV/CPC or shunt placement also suggests the absence of negative developmental sequelae secondary to fenestration of the third ventricular floor or cauterization of the choroid plexus. During CPC, we take great care to avoid the fornices, the choroidal fissure, and the ependymal surfaces. In both our North American and Ugandan experience, repeat ventriculoscopy for ETV failure after many months has revealed no residual evidence of the previous cauterization aside from the absence of choroid plexus, which has been converted to a line of scar tissue with little evidence of regeneration.

We have previously suggested that increased intraventricular pulsation amplitudes may be an important component of progressive ventriculomegaly, and that agerelated differences in ventricular compliance may underlie the decreased success for ETV in younger age groups if a component of the effect of ETV is to act as an additional pulsation absorber. ${ }^{14}$ Similarly, CPC may augment this effect of ETV by actually reducing the amplitude of intraventricular pulsations as demonstrated experimentally by Bering. ${ }^{1,14}$ Hydrocephalus arising from diffuse meningitis (as opposed to primary ventriculitis) may arise from a substantial reduction of the Windkessel mechanism for major arterial pulsations in the subarachnoid spaces, and could simply be more resistant to treatment by intraventricular pulsation reduction via ETV/CPC alone. Meningitis and cisternal scarring are both predictors of failure, possibly because subarachnoid space dysfunction may lead to greater increases in intraventricular pulsation amplitude and thus be the most difficult to overcome by ETV pulsation absorption (which is further reduced by cisternal obstruction) and pulsation reduction (CPC). This would be especially true in young infants for whom brain compliance is highest.

A recent retrospective analysis of hydrocephalus treatment for 5416 infants 1 year or younger at 41 children's hospitals in the Pediatric Health Information Systems database found a 1-year success rate of $35.5 \%$ for ETV alone and $60.4 \%$ for ventricular shunt placement. ${ }^{3}$
After controlling for prematurity, intraventricular hemorrhage, and MMC, ETV alone still had a higher risk of failure than shunt placement at 1 year (OR 2.6 [95\% CI 2.1-3.2]). This confirms our own experience, and that of others, that ETV alone fails in the majority of infants. ${ }^{13,15}$ By contrast, in the present series of 91 patients, 56 (62\%) required no further hydrocephalus-related procedures. Furthermore, 59 (65\%) were spared shunt dependence, which carries an average rate of about 3 additional operations for subsequent shunt malfunction per patient (177 total operations). ETV/CPC also carries a substantially lower risk of postoperative infection $(<1 \%)$ compared with shunt procedures, as demonstrated in the current study as well as in a larger previous study. ${ }^{13}$ In the past, and currently in many practices, all the patients in this study would have been treated primarily by shunt placement. If the North American shunt burden were reduced $50 \%$ by broadly adopting the strategy of primary treatment with ETV/CPC, the projected health care cost savings for the US could ultimately be on the order of $\$ 1$ billion annually. ${ }^{10}$ In this consecutive series of 100 nonselected patients, $59 \%$ were treated successfully without a shunt.

The present study suggests that the 1-year success for combined ETV/CPC as the primary treatment for hydrocephalus in infants is equivalent to shunt placement. Furthermore, given the ongoing risk of shunt malfunction throughout life, in contrast to successful treatment by ETV/CPC, avoiding shunt dependence from the beginning in more than half of infants may result in lifelong safety and quality of life benefits for the individual patients, and a reduction of the national health care cost burden. This, however, can only be determined by longterm, prospective multiinstitutional studies.

\section{Conclusions}

This series demonstrates the overall efficacy of ETV/ CPC as the primary and sole treatment for infant hydrocephalus in a North American population. The results are similar to those reported from Uganda and suggest that more than half of all infants presenting for initial treatment of hydrocephalus can be successfully treated in this way. Expectations should be tempered for those with hydrocephalus secondary to neonatal meningitis, those with prepontine cistern scarring, and those who have previously undergone shunt treatment. For all other infants, including those with so-called communicating hydrocephalus, 1-year success rates of roughly $60 \%$ and $80 \%$ would be expected for infants younger than and older than 6 months of age, respectively. Further studies will be necessary to validate these results in other centers and to investigate the longer-term consequences of treating infant hydrocephalus by ETV/CPC compared with shunt placement.

\section{Disclosure}

The authors report no conflict of interest concerning the materials or methods used in this study or the findings specified in this paper.

Author contributions to the study and manuscript preparation 
include the following. Conception and design: Warf. Acquisition of data: both authors. Analysis and interpretation of data: both authors. Drafting the article: both authors. Critically revising the article: both authors. Reviewed submitted version of manuscript: both authors. Approved the final version of the manuscript on behalf of both authors: Warf. Statistical analysis: Stone. Administrative/technical/ material support: Warf. Study supervision: Warf.

\section{References}

1. Bering EA Jr: Circulation of the cerebrospinal fluid. Demonstration of the choroid plexuses as the generator of the force for flow of fluid and ventricular enlargement. J Neurosurg 19:405-413, 1962

2. Chamiraju P, Bhatia S, Sandberg DI, Ragheb J: Endoscopic third ventriculostomy and choroid plexus cauterization in posthemorrhagic hydrocephalus of prematurity. Clinical article. J Neurosurg Pediatr 13:433-439, 2014

3. Jernigan SC, Berry JG, Graham DA, Goumnerova L: The comparative effectiveness of ventricular shunt placement versus endoscopic third ventriculostomy for initial treatment of hydrocephalus in infants. Clinical article. J Neurosurg Pediatr 13:295-300, 2014

4. Kiwanuka J, Bazira J, Mwanga J, Tumusiime D, Nyesigire E, Lwanga N, et al: The microbial spectrum of neonatal sepsis in Uganda: recovery of culturable bacteria in mother-infant pairs. PLoS ONE 8:e72775, 2013

5. Kulkarni AV, Drake JM, Mallucci CL, Sgouros S, Roth J, Constantini S, et al: Endoscopic third ventriculostomy in the treatment of childhood hydrocephalus. J Pediatr 155:254259.e1, 2009

6. Kulkarni AV, Warf BC, Drake JM, Mallucci CL, Sgouros S, Constantini S: Surgery for hydrocephalus in sub-Saharan Africa versus developed nations: a risk-adjusted comparison of outcome. Childs Nerv Syst 26:1711-1717, 2010

7. Li L, Padhi A, Ranjeva SL, Donaldson SC, Warf BC, Mugamba $\mathrm{J}$, et al: Association of bacteria with hydrocephalus in Ugandan infants. Clinical article. J Neurosurg Pediatr 7:73-87, 2011

8. Mandell JG, Kulkarni AV, Warf BC, Schiff SJ: Volumetric analysis in neurosurgery: II. Brain and CSF volumes discriminate neurocognitive outcomes in hydrocephalus. J Neurosurg Pediatr (in press)

9. Schiff SJ, Ranjeva SL, Sauer TD, Warf BC: Rainfall drives hydrocephalus in East Africa. Clinical article. J Neurosurg Pediatr 10:161-167, 2012

10. Simon TD, Riva-Cambrin J, Srivastava R, Bratton SL, Dean JM, Kestle JR: Hospital care for children with hydrocephalus in the United States: utilization, charges, comorbidities, and deaths. J Neurosurg Pediatr 1:131-137, 2008

11. Tamburrini G, Frassanito P, Iakovaki K, Pignotti F, Rendeli C, Murolo D, et al: Myelomeningocele: the management of the associated hydrocephalus. Childs Nerv Syst 29:1569-1579, 2013

12. Warf B, Ondoma S, Kulkarni A, Donnelly R, Ampeire M, Akona J, et al: Neurocognitive outcome and ventricular volume in children with myelomeningocele treated for hydrocephalus in Uganda. Clinical article. J Neurosurg Pediatr 4: 564-570, 2009

13. Warf BC: Comparison of endoscopic third ventriculostomy alone and combined with choroid plexus cauterization in infants younger than 1 year of age: a prospective study in 550 African children. J Neurosurg 103 (6 Suppl):475-481, 2005

14. Warf BC: Congenital idiopathic hydrocephalus of infancy: the results of treatment by endoscopic third ventriculostomy with or without choroid plexus cauterization and suggestions for how it works. Childs Nerv Syst 29:935-940, 2013

15. Warf BC: Hydrocephalus in Uganda: the predominance of infectious origin and primary management with endoscopic third ventriculostomy. J Neurosurg 102 (1 Suppl):1-15, 2005

16. Warf BC, Campbell JW: Combined endoscopic third ventriculostomy and choroid plexus cauterization as primary treatment of hydrocephalus for infants with myelomeningocele: long-term results of a prospective intent-to-treat study in 115 East African infants. Clinical article. J Neurosurg Pediatr 2:310-316, 2008

17. Warf BC, Campbell JW, Riddle E: Initial experience with combined endoscopic third ventriculostomy and choroid plexus cauterization for post-hemorrhagic hydrocephalus of prematurity: the importance of prepontine cistern status and the predictive value of FIESTA MRI imaging. Childs Nerv Syst 27:1063-1071, 2011

18. Warf BC, Dagi AR, Kaaya BN, Schiff SJ: Five-year survival and outcome of treatment for postinfectious hydrocephalus in Ugandan infants. Clinical article. J Neurosurg Pediatr 8:502508,2011

19. Warf BC, Dewan M, Mugamba J: Management of Dandy-Walker complex-associated infant hydrocephalus by combined endoscopic third ventriculostomy and choroid plexus cauterization. Clinical article. J Neurosurg Pediatr 8:377-383, 2011

20. Warf BC, Kulkarni AV: Intraoperative assessment of cerebral aqueduct patency and cisternal scarring: impact on success of endoscopic third ventriculostomy in 403 African children. Clinical article. J Neurosurg Pediatr 5:204-209, 2010

21. Warf BC, Mugamba J, Kulkarni AV: Endoscopic third ventriculostomy in the treatment of childhood hydrocephalus in Uganda: report of a scoring system that predicts success. Clinical article. J Neurosurg Pediatr 5:143-148, 2010

22. Warf BC, Stagno V, Mugamba J: Encephalocele in Uganda: ethnic distinctions in lesion location, endoscopic management of hydrocephalus, and survival in 110 consecutive children. Clinical article. J Neurosurg Pediatr 7:88-93, 2011

23. Warf BC, Tracy S, Mugamba J: Long-term outcome for endoscopic third ventriculostomy alone or in combination with choroid plexus cauterization for congenital aqueductal stenosis in African infants. Clinical article. J Neurosurg Pediatr 10:108-111, 2012

Manuscript submitted March 20, 2014.

Accepted July 29, 2014.

Please include this information when citing this paper: published online August 29, 2014; DOI: 10.3171/2014.7.PEDS14152.

Address correspondence to: Benjamin C. Warf, M.D., Department of Neurosurgery, Children's Hospital Boston, 300 Longwood Ave., Boston, MA 02115. email: benjamin.warf@childrens.harvard. edu. 